\title{
Salvage of Failed Osteoarticular Tibia Allografts with Knee Arthroplasties
}

\author{
G. Ulrich Exner'1, Pascal A. Schai ${ }^{2}$, Tobias C. Bühler ${ }^{*}$, Theodore I. Malinin ${ }^{4}$ \\ ${ }^{1}$ Orthopaedie Zentrum Zuerich, Zuerich, Switzerland \\ ${ }^{2}$ Luzerner Kantonsspital Wolhusen, Wolhusen, Switzerland \\ ${ }^{3}$ Kantonsspital Baden AG, Baden, Switzerland \\ ${ }^{4}$ Department of Orthopaedics, University of Miami Miller School of Medicine, Miami, USA \\ Email: *exner@orthopaedie-zuerich.ch
}

How to cite this paper: Exner, G.U., Schai, P.A., Bühler, T.C. and Malinin, T.I. (2020) Salvage of Failed Osteoarticular Tibia Allografts with Knee Arthroplasties. Open Journal of Orthopedics, 10, 67-76.

https://doi.org/10.4236/ojo.2020.104009

Received: March 3, 2020

Accepted: April 12, 2020

Published: April 15, 2020

Copyright (C) 2020 by author(s) and Scientific Research Publishing Inc. This work is licensed under the Creative Commons Attribution-NonCommercial International License (CC BY-NC 4.0).

http://creativecommons.org/licenses/by-nc/4.0/

\begin{abstract}
Background: Limb preservation in musculoskeletal tumor surgery has largely replaced amputation. Biologic reconstructions are now performed as preferred choice; if not feasible options are "megaprostheses", allografts or composites. Endoprosthetic reconstructions usually provide immediate function, but fail at long term. Osteochondral allografts allow for one-to-one restoration and have potential for incorporation; however degeneration of the cartilage requiring revision almost inevitably will occur. In most cases, revision is then done by endoprosthetic replacement. Aim: In our patients, resurfacing of retained allografts failed. Problems encountered are presented and solutions proposed. Case Presentation: Resurfacing over retained allografts in the 2 index cases has resulted in failures related to fractures and instability. Revision with massive constrained endoprostheses was needed. Based on the experience with these failures, primary endoprosthetic replacement anchored in vital bone in a following case resulted in stable function. Conclusion: Knee replacement for advanced degeneration of the osteochondral allograft apparently needs choosing increased femoro-tibial constraint systems and stem extensions anchored to vital host bone.
\end{abstract}

\section{Keywords}

Tumor, Allograft, Arthroplasty

\section{Introduction}

Allografts allow us to reconstruct defects of tumor resection one-to-one and are therefore especially an option to be considered in growing children and young adults, preserving durable limb function [1]. However, a high failure rate is re- 
lated to fracture, infection and degeneration of the osteoarticular region.

Toy et al. [2] and Verbeek et al. [3] addressed problems encountered in femoral osteoarticular allografts and complications in revision to total hip replacement (THA) or total knee replacement (TKA); 61\% of THA/TKA respectively in retained allografts had complications, mostly structural failures (periprosthetic fractures/allograft fractures [3]). A study comparing outcome of proximal tibia reconstructions with either osteoarticular allografts or endoprosthetic replacement showed separate advantages, however not significantly different overall failure rates (at 10 years $44 \%$ for endoprostheses and 32\% for allografts [4]).

Details of endoprosthetic salvage procedures of failed allograft reconstructions have been sparsely described [5]. We wish to report on failures experienced with resurfacing arthroplasty for articular degeneration on retained tibial osteochondral allografts. The observations generally lead us to anchor endoprostheses directly to vital autochthonous bone of the patients.

\section{Patients and Methods}

Three patients received arthroplasties for failed osteoarticular reconstructions after resection of osteosarcoma.

\section{Details of the Patients}

\section{Case 1}

The female patient (C.D. $\left.{ }^{\star} 1979\right)$ received osteoarticular allograft reconstruction for osteosarcoma of her right proximal tibia at 13 years of age (04/1992). After corrective osteotomy (09/1993) and operative elevation of the lateral tibial plateau depression (05/1994), exchange of the tibial allograft became necessary for tibial plateau fracture (05/1996). Because of subchondral fracture of the replaced osteoarticular allograft 6.5 years later (Figure 1(A)-(a), Figure 1(A)-(b)), nonconstrained bicompartmental resurfacing knee arthroplasty was performed (10/2002, Natural Knee II), cemented anchoring the tibial component within the tibial allograft (Figure 1(B)-(a)). Fracture through a screw hole of the former plate three years after knee arthroplasty initiated reosteosynthesis with angular stable plate (08/2005) aiming at preserving the knee arthroplasty and allograft reconstruction (Figure 1(B)-(b)). Increasing instability led to allograft explantation and implantation of the hinged tumor prosthesis system MUTARS, anchoring femoral and tibial shaft extensions within vital host bone maintaining the preserved allograft tibial tuberosity (11/2005). Later traumatic patella fracture $(01 / 2016)$ was treated by osteosynthesis, fortunately not interfering with the well functioning prosthesis at last follow-up (10/2018; Figures $1(C)-[(a)-(d)])$.

\section{Case 2}

The male patient (M.F. ${ }^{\star} 1975$ ) had osteoarticular allograft reconstruction for osteosarcoma of his right proximal tibia at 17 years of age (10/1992). Fracture of the tibial allograft three years later was treated by exchanging with a new allograft 


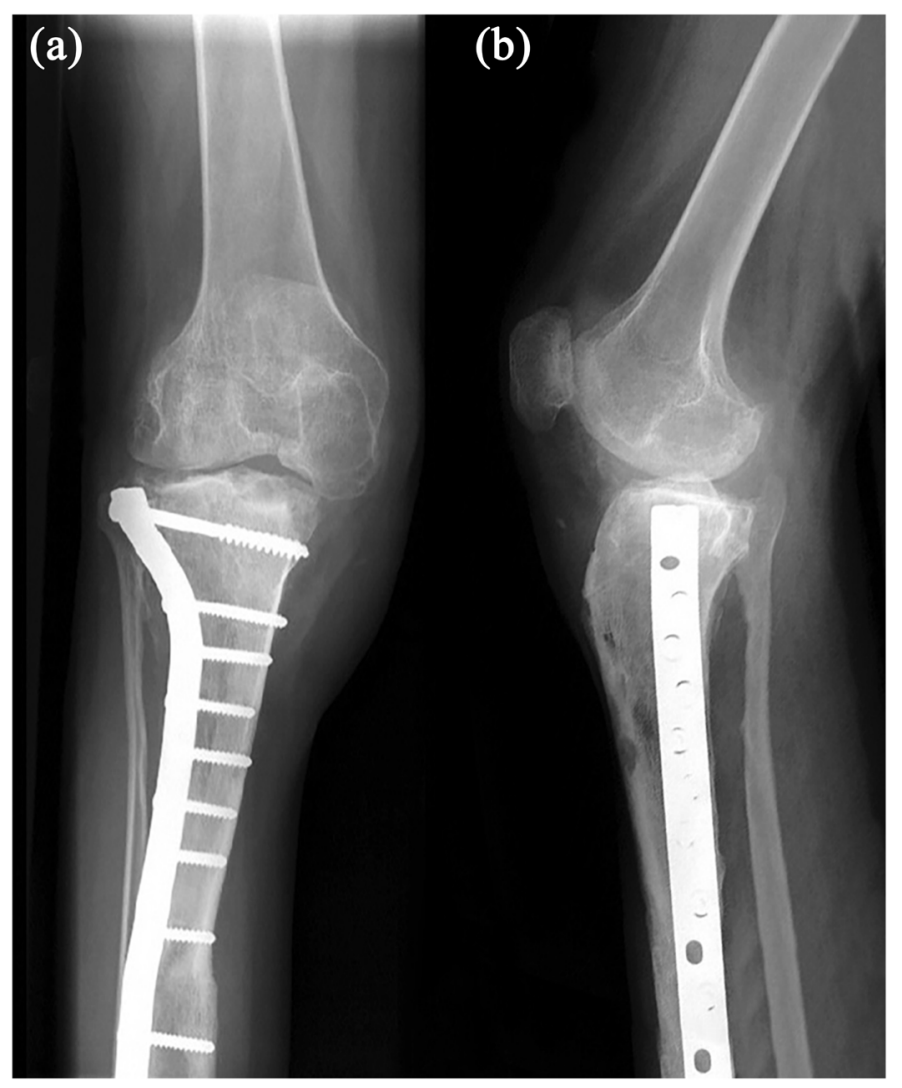

(A)

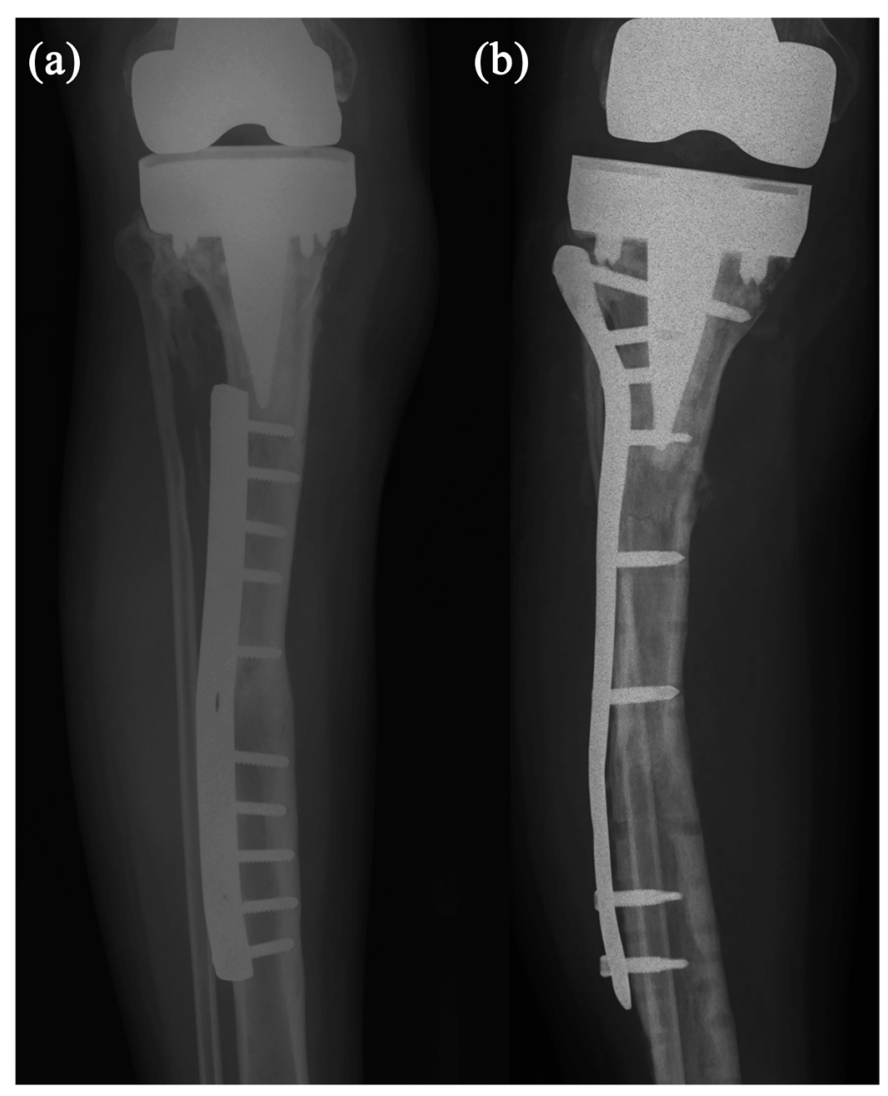

(B) 


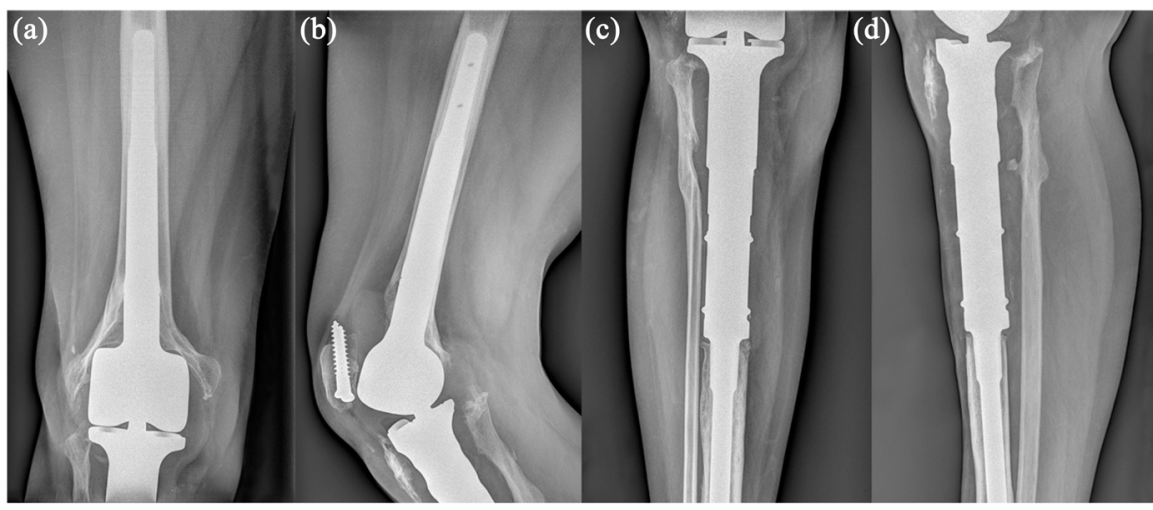

(C)

Figure 1. (A) Cartilage degenerations and subchondral fractures of the replaced osteoarticular allograft depicting knee arthritis involving all compartments (10/2002). (a) Frontal radiograph; (b) Lateral radiograph. (B) Radiographic aspects after knee arthroplasty preserving the knee arthroplasty and allograft reconstruction. (a) Frontal tibial radiograph after resurfacing knee arthroplasty anchored within tibial allograft (10/2002); (b) Progressive angular deformity through fatigue of the allograft and tibial insert polyethylene wear (08/2005). (C) 13 years (10/2018) after revision knee arthroplasty with MUTARS components anchored to host bone (2 years after patella fracture 01/2016). (a) Frontal radiograph of femoral component; (b) Lateral radiograph of femoral component; (c) Frontal radiograph of tibial component; (d) Lateral radiograph of tibial component.

(Figure 2(A)-(a) (11/1995)/Figure 2(A)-(b) (02/1996)). After developing degenerative changes bicompartmental knee arthroplasty using non-constrained resurfacing components was performed (03/2003), anchoring the tibial component within the tibial allograft. Early aseptic loosening one year later required exchange with a stemmed component bridging the allograft-tibia junction and medial collateral ligament reconstruction (Figure 2(A)-(c) (11/2004)/Figure 2(A)-(d) (02/2005)). Tibiofemoral instability over the follwing years made revision knee arthroplasty necessary, implanting the hinged tumor prosthesis system MUTARS, anchoring noncemented femoral and tibial shaft extensions within vital host bone (09/2009). Because of later aseptic loosening femoral component exchange using a custom made metaphyseal sleeve was performed (08/2015; Figure 2(B)-(a)/Figure 2(B)-(b)). At present, the patient is highly satisfied because of his stable leg function not experienced before (03/2019; Figures $2(\mathrm{C})-[(\mathrm{a})-(\mathrm{d})])$.

\section{Case 3}

The male patient (T.M. $\left.{ }^{*} 1968\right)$ had hemicondylar osteoarticular allograft reconstruction for osteosarcoma of his left medial tibial condyle (12/1992; Figure 3(A)-(a)/Figure 3(A)-(b)). Subchondral fracture of the allograft was successfully treated by allograft tibial plateau elevation and interposition of autologous iliac bone graft (12/1994; Figure 3(A)-(c)/Figure 3(A)-(d)). Because of progressive degenerative changes with endstage knee arthritis the patient asked for knee arthroplasty (Figure 3(B)-(a)/Figure 3(B)-(b)). Based upon the observations in the former two patients, the MUTARS tumor prosthesis was implanted 


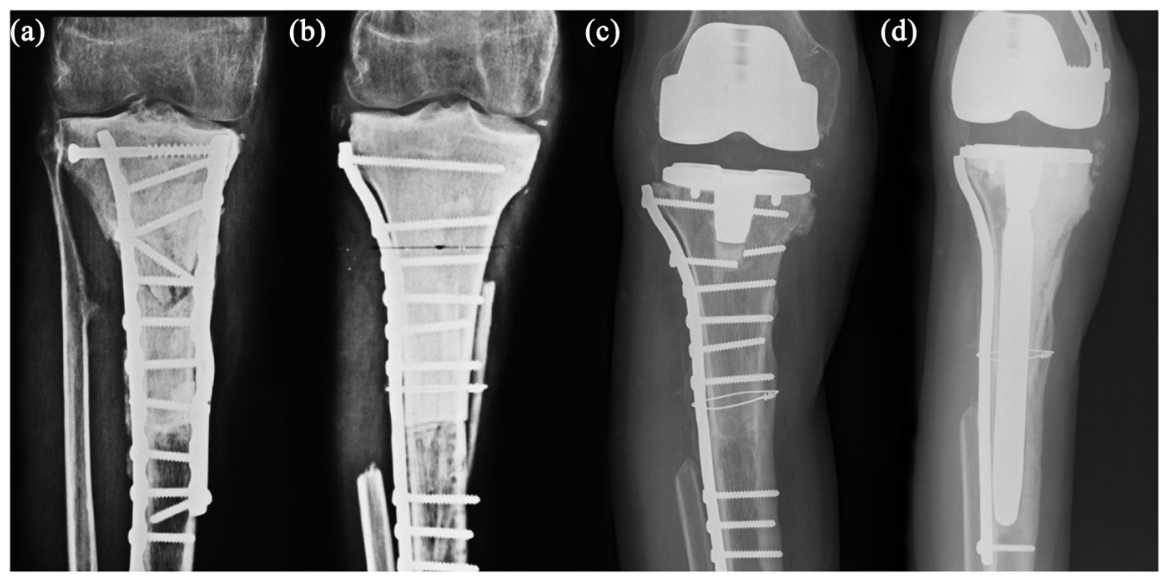

(A)

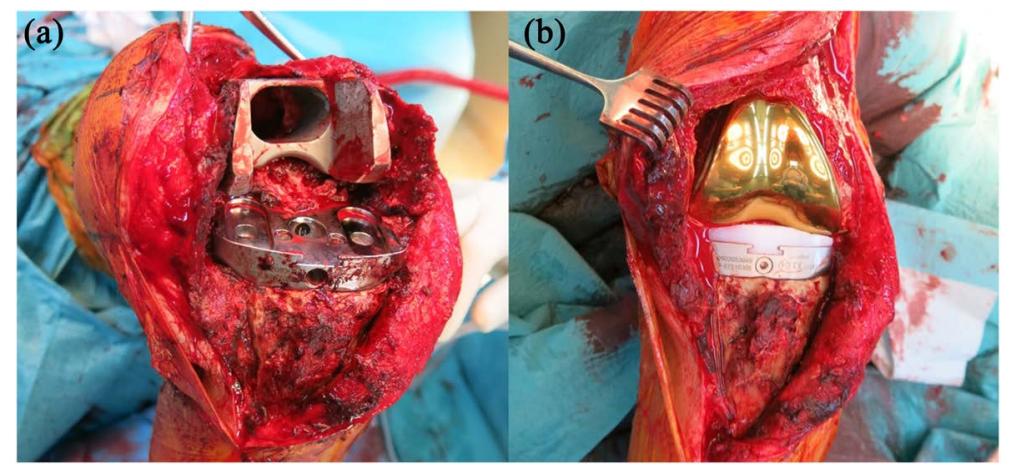

(B)

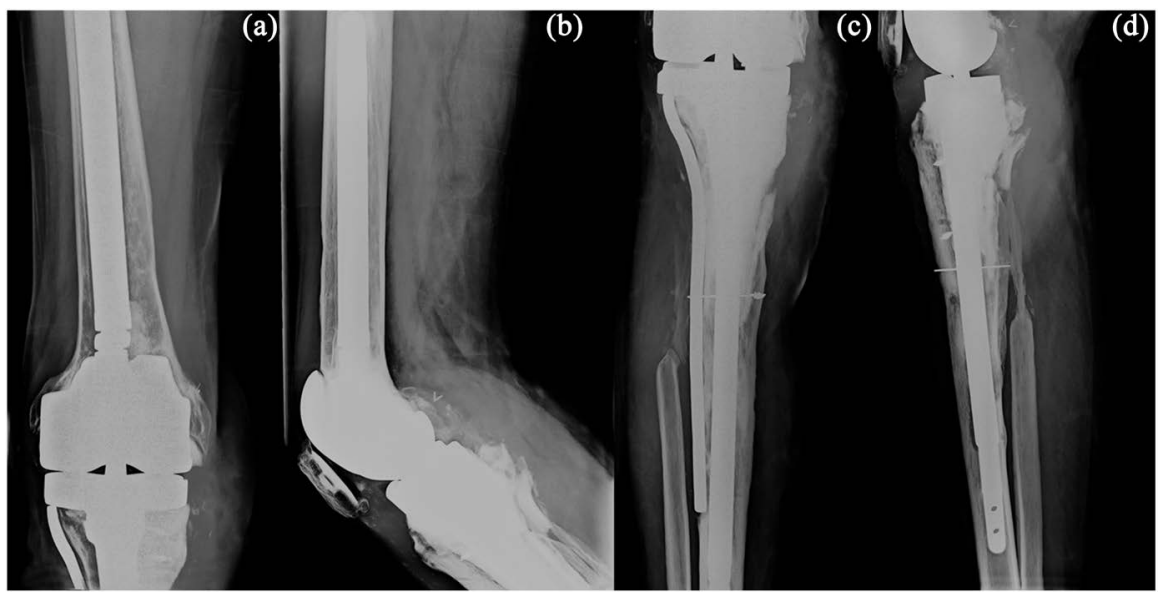

(C)

Figure 2. (A) Sequelae of tibial allograft reconstruction and knee arthroplasty. (a) Failed first osteochondral tibial allograft for fracture at the allograft-host junction (11/1995); (b) Frontal radiograph after replacement allograft reconstruction (02/1996); (c) Aseptic tibial component loosening of the resurfacing knee arthroplasty (11/2004); (d) Frontal radiograph after exchange with stemmed component (02/2005). (B) Intraoperative sequences of knee arthroplasty (08/2015). (a) Custom made metaphyseal spacer implanted; (b) Frontal aspect after implantation of MUTARS total knee arthroplasty. (C) 3.5 years after MUTARS revision total knee arthroplasty with custom made femoral sleeve (03/2019). (a) Frontal radiograph of femoral component; (b) Lateral radiograph of femoral component; (c) Frontal radiograph of tibial component; (d) Lateral radiograph of tibial component. 


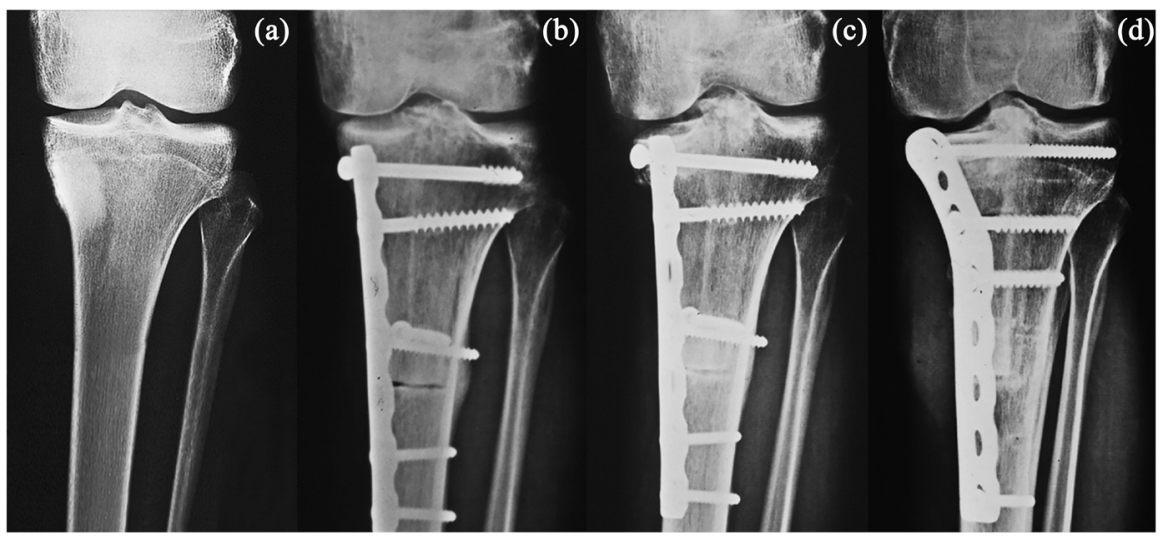

(A)

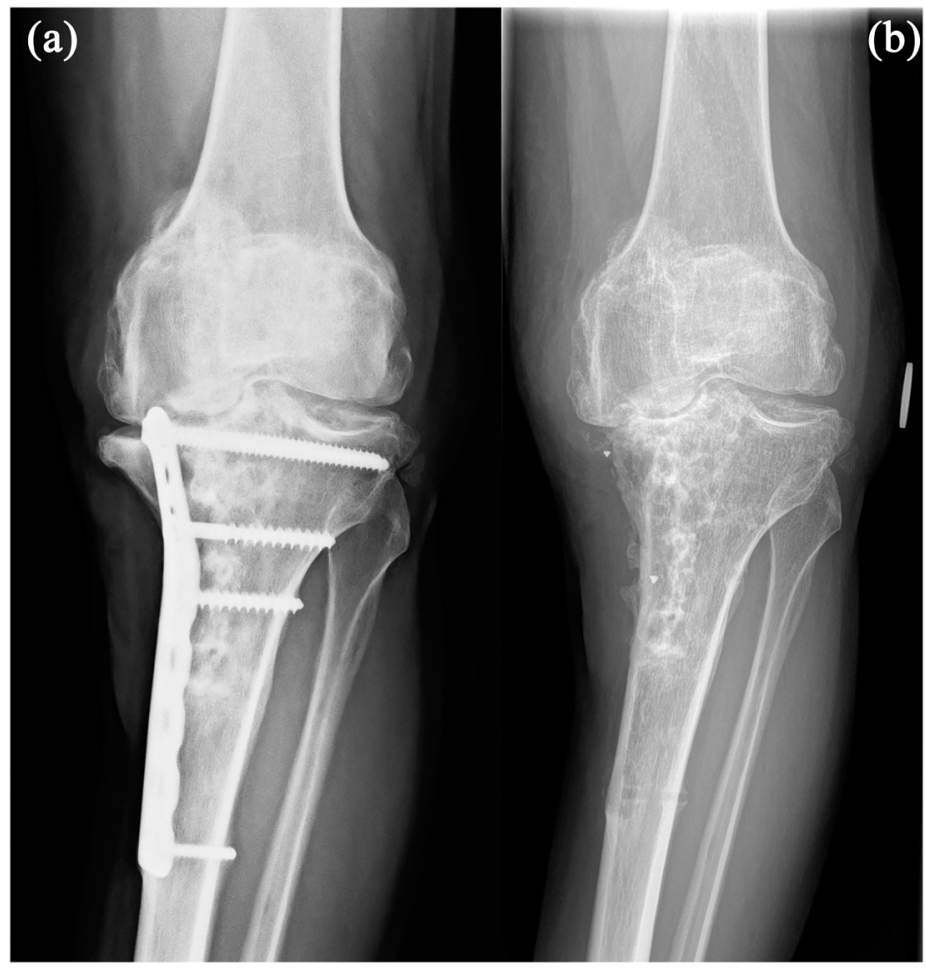

(B)

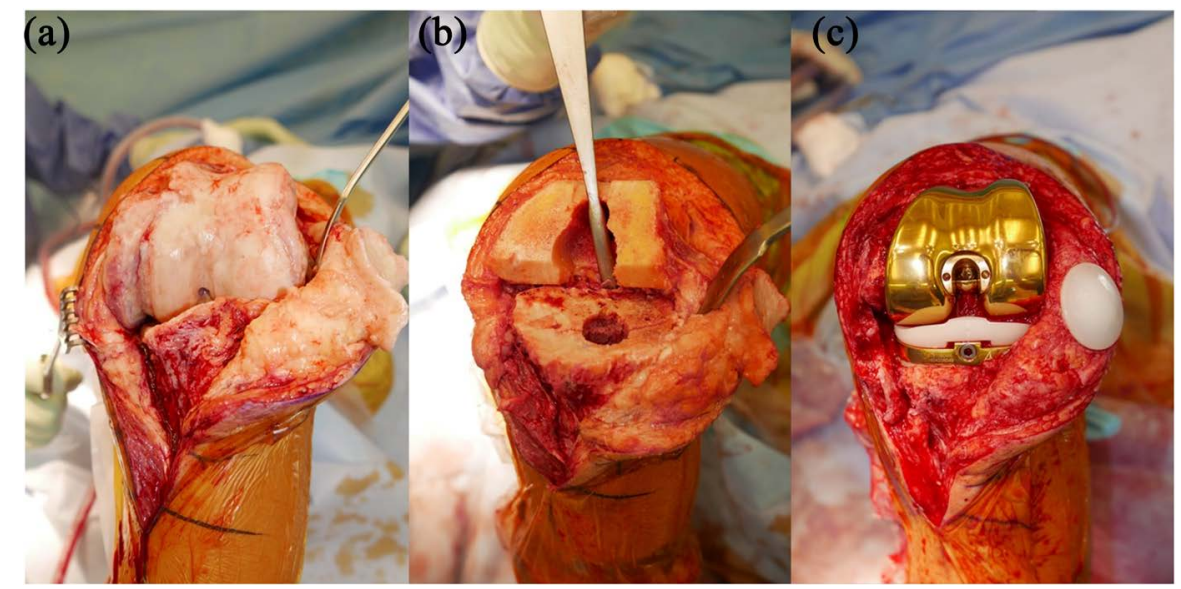

(C) 


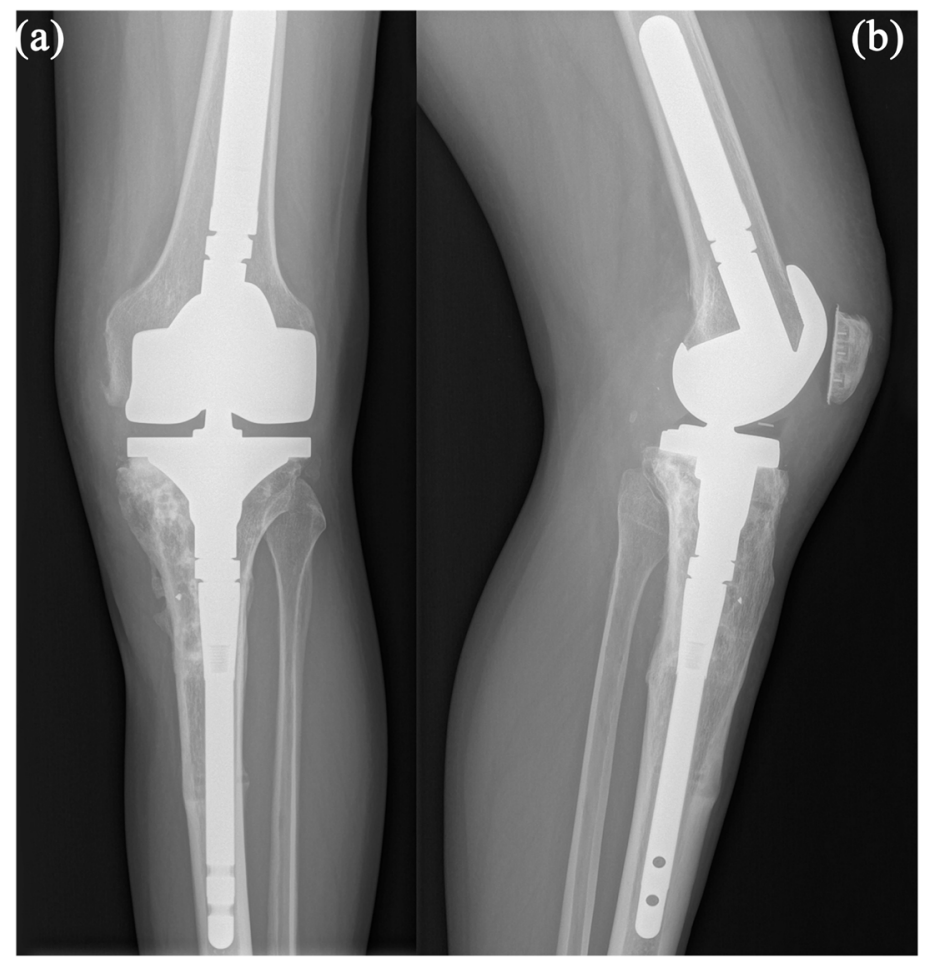

(D)

Figure 3. (A) Sequences of tibial allograft reconstruction for osteosarcoma. (a) Osteosarcoma of left medial tibial head (12/1992); (b) Hemicondylar osteoarticular allograft reconstruction (12/1992); (c) Subchondral fracture of the tibial allograft (12/1994); (d) Allograft tibial plateau elevation and interposition of autologous iliac bone graft (12/1994). (B) Endstage left knee arthritis (08/2016). (a) Collapse of osteochondral medial tibial allograft and consecutive degenerative knee arthritis involving all compartments; (b) Frontal radiograph after implant removal before knee arthroplasty. (C) Intraoperative sequences of knee arthroplasty (12/2016). (a) Frontal view of degenerative knee arthritis; (b) Bone cuts performed, revealing residual minor bone defect of medial tibial condyle; (c) Frontal aspect after implantation of MUTARS total knee arthroplasty. (D) Radiographic documentation at 2 years follow-up time (12/2018). (a) Frontal radiograph of MUTARS left total knee arthroplasty; (b) Lateral view of MUTARS left total knee arthroplasty.

(Figures 3(C)-[(a)-(c)]), supplying immediate stable knee function by means of the rotation hinge coupling and anchoring the arthroplastic components using noncemented stems within vital host bone (12/2018; Figure 3(D)-(a)/Figure $3(D)-(b))$. At present (03/2020), the patient is satisfied with unlimited physical activities including downhill skiing.

\section{Results Summarized}

Three patients with osteoarticular allograft replacement of the proximal tibia needed knee arthroplasty for cartilage degeneration 10, 11, and 24 years after the initial treatment for proximal tibia osteosarcomas.

Two of them were initially treated with standard resurfacing knee arthroplasties that failed within three years due to fracture of the allograft and instability of 
the non-hinged joints. They were revised to tumor reconstruction systems (MUTARS, Implantcast) with fully rotating hinged knee replacements resulting in stable well functioning legs.

In a third patient treated with a hemicondylar osteoarticular allograft needing knee replacement 24 years after initial reconstruction, based on the experience with the patients reported before, a fully constrained resurfacing knee arthroplasty system with stem extensions was implanted giving initial stable contact to the vital host bone.

\section{Discussion}

Whether it is preferable to perform reconstruction of large segmental bone defects after tumor resections including joints with allografts or with massive endoprostheses is still unsolved. All-over results are comparable, however there are differences with regard to causes of failure [3] [4]. Failures of osteoarticular allografts are primarily caused by fractures and degeneration of the articular cartilage.

In the presented two index cases ( 1 and 2), fractures of the allografts were addressed by osteosynthesis and/or replacement respectively. Later progressive degeneration of the osteoarticular component was initially treated by resurfacing types of endoprostheses on the retained allograft. At long term, these revisions also failed because of new fractures of the massive allograft and/or instability of the non-hinged joints. Finally, the failed reconstructions over the retained allografts were revised with hinged endoprostheses anchored in vital autochthonous bone. Following these revisions both patients perceived significant functional improvement with full weight bearing and improved stability by the hinged joints. Integrating the experience from the first two cases, the MUTARS tumor prosthesis was implanted in our third patient (case 3) as the first line knee arthroplasty system, supplying stable knee function by means of the rotation hinge coupling and anchoring the arthroplastic components using noncemented stems within vital host bone.

Tibial allograft reconstructions after resection of malignant bone tumors have become standard of care to preserve limb function in skeletally immature growing children, especially to preserve the opposite growth plate, and in young adults [1].

Proximal tibia reconstruction after bone tumor resection with osteoarticular allografts yielded similar failures compared to endoprosthetic replacement, favouring allograft reconstructions in younger patients to achieve better extensor mechanism function [4].

In patients requiring osteoarticular tibial allografts to preserve the knee joint, the articular surface may over time develop secondary degenerative arthritic changes, inevitably needing knee replacement surgery [2].

Successful salvage of failed osteoarticular allografts was reported after endoprosthetic revisions preserving limb function (80\% salvage of primary recon- 
struction at 160 months followup time [6]).

Treating patients with osteoarticular femoral allografts and subsequent degenerative bone disease showed comparable outcome with total knee arthroplasty in retained femoral allografts, endoprosthesis after allograft removal or primary allograft-prosthesis composites [3].

In their series of 26 distal femoral osteoarticular allografts [2], of seven conversions to allograft-prosthetic composites for progressive joint degeneration, four had subsequent allograft fractures or relevant soft-tissue related problems.

Apart of chemotherapy, gender and tumor grade as risk factors for knee arthroplasties after osteoarticular allograft reconstruction about the knee joint, the arthroplasty system was found to be an important factor for component survival and need for revision knee arthroplasty [7]. All of the patients with non-stemmed components suffered major complications, with significant rate of fractures in unsupported regions of retained allografts, requiring operative reinterventions.

Reporting on total knee replacement in a series of 35 previous recipients of fresh osteochondral allograft transplants, a non-constrained knee arthroplasty system was used, only using stemmed components in three cases [8]. These patients did not have complete tibial plateau allograft replacements and therefore cannot be compared to our cases. According to the low number of stem extensions, $17 \%$ of the knees required revision knee arthroplasty within the mean follow-up time of 7.5 years because of aseptic loosening.

The MUTARS modular endoprosthesis system has proven a reliable option for primary knee replacement in tumor resection, with durable fixation of the components over midterm follow-up time (89\% retention of the implant over 8.9 years [9]). Various surgical difficulties were encountered for limb preserving salvage using the modular MUTARS system even when completely resecting the original allograft due to altered soft tissue and remnant bone structures, yet with reasonable endoprosthesis survival time [5].

Further data hopefully will improve the concept of maximally bone preserving endoprosthetic replacement following failure of the articular component of massive allografts.

\section{Conclusions}

From our experience with massive allografts, we would reserve replacement of the proximal tibia with osteoarticular allografts primarily for very young patients in their growing age. Replacement by allograft versus endoprosthesis as initial procedure for reconstructions has to be carefully weighed against either system.

Patients of younger age with osteochondral allograft reconstruction for tumor resection of the proximal tibia will inevitably develop arthritic degeneration of the knee joint, eventually requiring prosthetic knee replacement. Standard resurfacing knee arthroplasty systems after osteochondral allograft reconstructions appear prone to failure, either for early loosening of the arthroplastic component fixed onto the retained osteoarticular allografts or for sequential instability of the 
tibiofemoral articulation. Therefore knee replacement for advanced knee arthritis under these conditions demands a knee arthroplasty system with increased tibiofemoral constraint to compensate for the weakened ligament structures and stem extensions anchored to vital host bone.

\section{Conflicts of Interest}

The authors declare no conflicts of interest regarding the publication of this paper.

\section{References}

[1] Ramseier, L.E., Malinin, T.I., Temple, H.T., Mnaymneh, W.A. and Exner, G.U. (2006) Allograft Reconstruction for Bone Sarcoma of the Tibia in the Growing Child. The Journal of Bone \& Joint Surgery (Br), 88-B, 95-99.

https://doi.org/10.1302/0301-620X.88B1.16253

[2] Toy, P.C., White, J.R., Scarborough, M.T., Enneking, W.F. and Gibbs, C.P. (2010) Distal Femoral Osteoarticular Allografts. Long-Term Survival, But Frequent Complications. Clinical Orthopaedics and Related Research, 468, 2914-2923. https://doi.org/10.1007/s11999-010-1470-x

[3] Verbeek, B.M., Kaiser, C.L., Pereira, N.R.P., et al. (2017) Primary Arthroplasty in Healed Osteoarticular Allograft in Patients with History of Primary Femoral Bone Tumors. Surgical Oncology, 26, 498-505. https://doi.org/10.1016/j.suronc.2017.10.003

[4] Albergo, J.I., Gaston, C.L., Aponte-Tinao, L.A., et al. (2017) Proximal Tibia Reconstruction after Bone Tumor Resection: Are Survivorship and Outcomes of Endoprosthetic Replacement and Osteoarticular Allograft Similar? Clinical Orthopaedics and Related Research, 475, 676-682. https://doi.org/10.1007/s11999-016-4843-y

[5] Foo, L.S.S., Hardes, J., Henrichs, M., Ahrens, H., et al. (2011) Surgical Difficulties Encountered with Use of Modular Endoprosthesis for Limb Preserving Salvage of Failed Allograft Reconstruction after Malignant Tumor Resection. The Journal of Arthroplasty, 26, 744-750. https://doi.org/10.1016/j.arth.2010.06.011

[6] Wang, J., Temple, H.T., Pitcher, J.D., et al. (2006) Salvage of Failed Massive Allograft Reconstruction with Endoprosthesis. Clinical Orthopaedics and Related Research, 443, 296-301. https://doi.org/10.1097/01.blo.0000194071.40892.ab

[7] DeGroot, H. and Mankin, H. (2000) Total Knee Arthroplasty in Patients Who Have Massive Osteoarticular Allografts. Clinical Orthopaedics and Related Research, 373, 62-72. https://doi.org/10.1097/00003086-200004000-00009

[8] Morag, G., Kulidjian, A., Zalzal, P., et al. (2006) Total Knee Replacement in Previous Recipients of Fresh Osteochondral Allograft Transplants. The Journal of Bone \& Joint Surgery, 88, 541-546. https://doi.org/10.2106/00004623-200603000-00012

[9] Bus, M.P.A., van de Sande, M.A.J., Fiocco, M., et al. (2017) What Are the LongTerm Results of MUTARS Modular Endoprostheses for Reconstruction of Tumor Resection of the Distal Femur and Proximal Tibia? Clinical Orthopaedics and Related Research, 475, 708-718. https://doi.org/10.1007/s11999-015-4644-8 